\title{
The Role of Dynamic ctDNA Monitoring During Combination Therapies of BRAF V600E-Mutated Metastatic Colorectal Cancer: A Case Report
}

This article was published in the following Dove Press journal: OncoTargets and Therapy

\author{
Zhan Wang ${ }^{l, *}$ \\ Chen-Yang Ye $\mathbb{1 D}^{\mathrm{I}}{ }^{*}$ \\ Wen-Li Zhou' \\ Miao-Miao Wang' \\ Wei-Ping Dai' \\ Jingjing Zheng ${ }^{2}$ \\ Yuan-Sheng Zang' \\ 'Department of Medical Oncology, \\ Changzheng Hospital, Naval Medical \\ University, Shanghai 200003, People's \\ Republic of China; ${ }^{2}$ Burning Rock \\ Biotech, Guangzhou, People's Republic of \\ China
}

*These authors contributed equally to this work
Background: BRAF V600E mutation represents a group of colorectal carcinoma with poor prognosis. Although treatment strategies have been recommended after clinical investigations, the progression-free survival is short and unsatisfying.

Case Presentation: Here, we present the case of a 28 -year-old male diagnosed with ascending colon adenocarcinoma with multiple liver metastases. Treatment with FOLFIRI plus cetuximab and vemurafenib achieved partial response, following which the patient received conversion surgery with clear resection margin. After disease recurrence, he received combination treatment of nivolumab and regorafenib. Until August 2020, the patient achieved a partial response with more than 12 months progression-free survival. Circulating tumor DNA (ctDNA) was monitored during the patient's treatment. His ctDNA fractions exhibited significant elevation two months before disease progression. As a comparison, the tumor markers were not elevated until the patient was confirmed PD through CT imaging.

Conclusion: This case exemplifies how liquid biopsy and ctDNA sequencing can aid in real-time molecular characterization of tumors.

Keywords: colorectal, BRAF V600E, ctDNA monitoring, carcinoma

\section{Case Presentation}

A 28-year-old man was examined for suffering abdominal pain for 6 months, otherwise he was fit and well, with no significant comorbid medical history. He was diagnosed with ascending colon adenocarcinoma in October 2017 with multiple metastatic lesions in the liver. Lung lesion was noted through chest computed tomography (CT) and was confirmed to be a colorectal liver metastasis (CRLM). Further testing identified a BRAF V600E mutation and microsatellite stable (MSS) in his tumor, via next-generation sequencing (NGS). He received first line FOLFOXIRI plus bevacizumab treatment (Irinotecan $270 \mathrm{mg} \mathrm{d} 1+$ oxaliplatin $150 \mathrm{mg} \mathrm{d} 2+\mathrm{CF} 0.74 \mathrm{~g}$ ivgtt $\mathrm{d} 1+5$-FU $0.74 \mathrm{~g}$ iv $\mathrm{d} 1,2.2 \mathrm{~g}$ civ $48 \mathrm{~h}, \mathrm{q} 2 \mathrm{w}$, bevacizumab $400 \mathrm{mg} / \mathrm{cycle}$ ) from December 2017. The best response was partial response (PR) with tumor remission in both of his liver and lung lesions. He was found to have disease progression in May 2018 with increased liver metastatic lesion size. He went to our hospital for further treatment. Considering the BRAF V600E mutation, FOLFIRI plus cetuximab and vemurafenib (FIVC, NCT03727763) (irinotecan 320mg ivgtt d1 +CF 0.75g ivgtt $\mathrm{d} 1+5-\mathrm{Fu} 0.75 \mathrm{~g}$ iv d1, 4.25g civ 46h + cetuximab 900mg ivgtt d1 q2w + vemurafenib
Correspondence: Yuan-Sheng Zang Department of Medical Oncology, Chang Zheng Hospital, Naval Medical University, No. 4I5, Feng Yang Road, Shanghai 200003, People's Republic of China Tel +86-I38I6584620

Email doctorzangys@163.com 
$960 \mathrm{mg} 2 /$ day) was used as second-line treatment. Efficacy of treatment was evaluated every 3-months through CT imaging. Tumor markers in blood were examined every month. After 5 cycles of treatment, liver lesions were significantly decreased and radiograph revealed PR (Figure 1). CEA measurement decreased from $100 \mathrm{ng} / \mathrm{mL}$ to $30 \mathrm{ng} / \mathrm{mL}$ (Figure 2). He underwent conversion surgery with colectomy, hepatectomy and cholecystotomy in September 2018. Genomic sequencing using the patient's tumor tissue sample and blood sample confirmed the BRAF V600E mutation as one major oncogenic molecular alteration in his tumor. Blood ctDNA detection was planned to monitor the patient's molecular disease status after a 3-month interval.

The patient subsequently received adjuvant therapy with FIVC for 7 cycles from November 2018 to February 2019. Two cycles of maintenance therapy were conducted with capecitabine (1.5g bid d1-14) and bevacizumab (500mg d1 q3w) in May 2019 (Figure 1). However, CT scanning revealed an abdominal disease progression in liver in late June (Figure 3). According to his ctDNA results in April, BRAF V600E was the main tumor genomic alteration detected. Therefore, FIVC treatment was used again for two cycles, unfortunately, no clinical improvement was observed. His disease continued slow progression with increased liver lesion. Tumor genetic testing using NGSbased ctDNA sequencing also revealed increased mutation fraction in blood samples. The patient was subsequently treated with nivolumab (200mg ivgtt d1 q2w) plus regorafenib (80mg qd) from August 2019 [NCT04110093]. Until late August 2020, the patient's disease evaluation was PR with more than 12-month PFS (Figure 3). The patient gave written informed consent for publication of his case. The case details were approved for publication by the ethical committee of Changzheng Hospital.

\section{Discussion and Conclusions}

Colorectal cancer (CRC) is one of the most frequent cancers worldwide. ${ }^{1}$ The BRAF V600E mutation is a known oncogenic driver and is present in approximately $10 \%$ of CRC. $^{2}$ The V600E mutation in BRAF leads to activation of the MAPK signaling pathway, contributing to the progression of CRC. Such mutation defines a specific subgroup of CRC with poor prognosis. ${ }^{3,4}$ Current NCCN guideline recommended combination treatment of

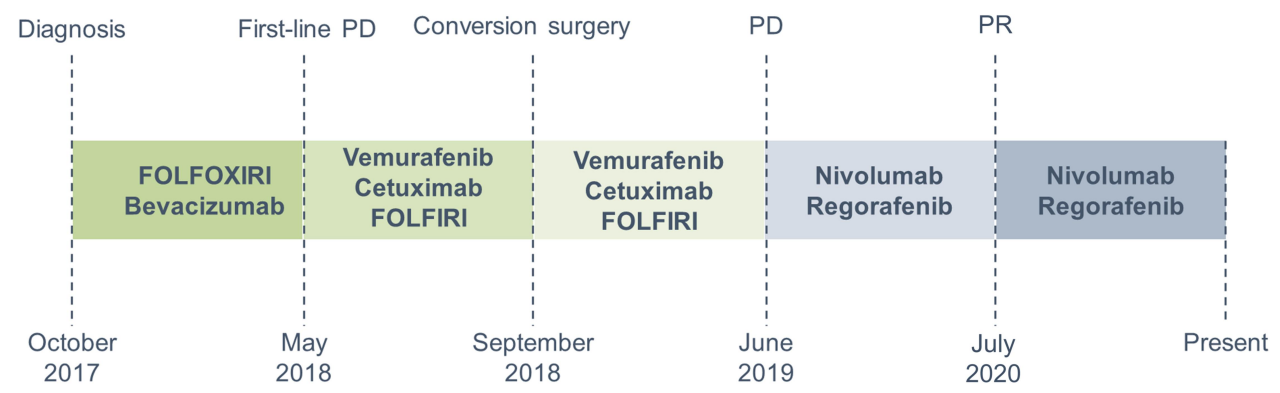

Figure I Clinical history of the patient. Treatment and molecular testing is indicated in the figure.

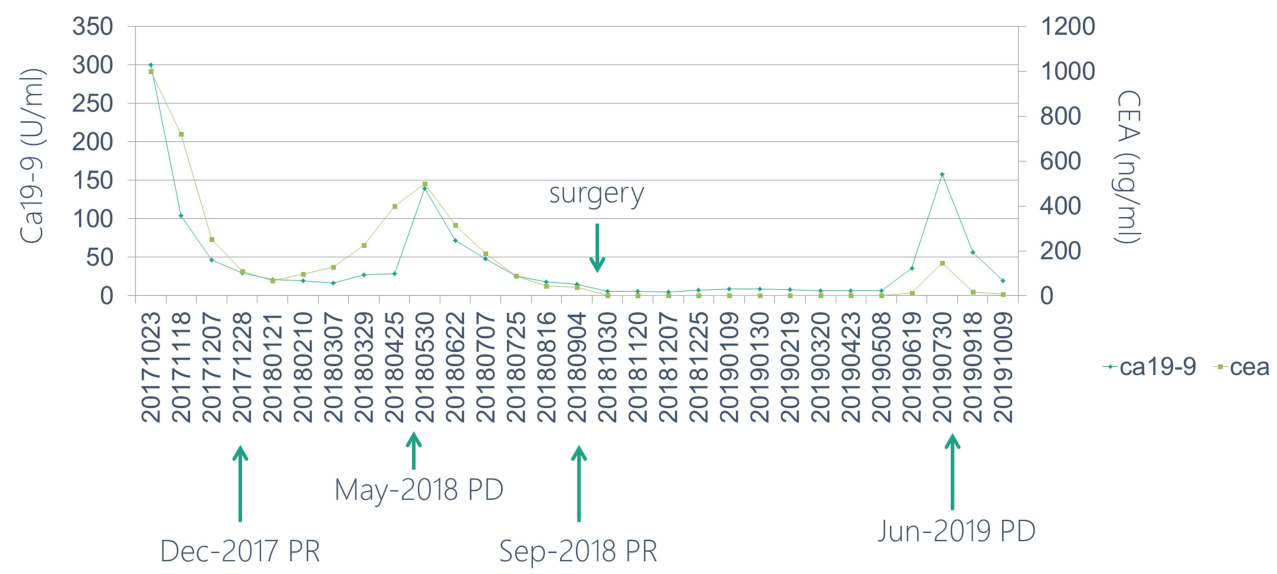

Figure 2 Tumor marker levels of the patient during treatment. 
June-2019
Disease progression

December-2019

Stable Disease

July-2020

Partial Response
A1

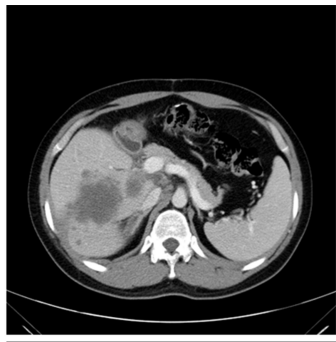

B1

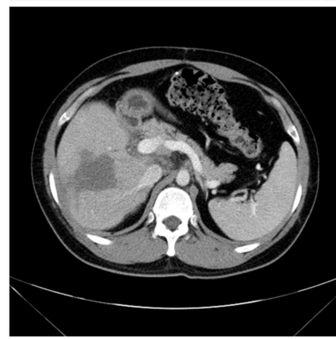

B2
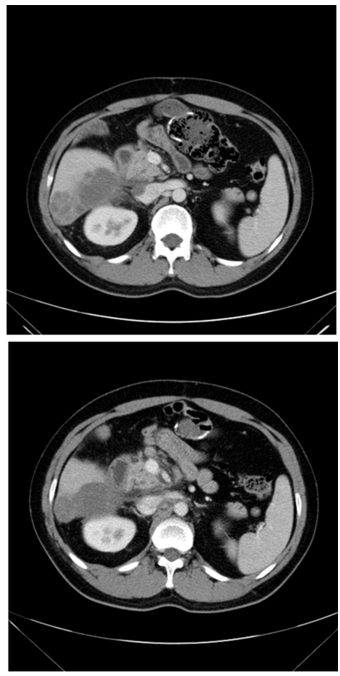

C1
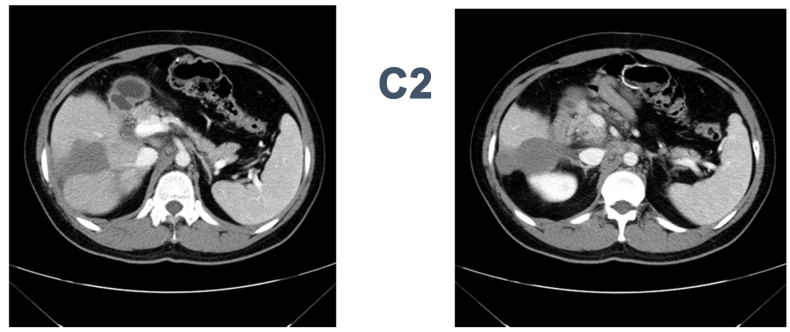

Figure 3 CT images of the patient before $(\mathbf{A})$ and after (B and $\mathbf{C})$ the regorafenib and nivolumab combination treatment.

encorafenib, cetuximab/panitumumab in second-line setting of BRAF V600E positive $\mathrm{mCRC}$, while the BRAF inhibitor is not available in China right now. Other treatment strategies are under investigation, such as the FIVC strategy. Although these combinations achieved tumor responses, as far as we know, there was no conversion surgery reported following such treatment. The present case showed complete success for a R0 resection following the targeted combination therapy.

Molecular changes identified through ctDNA have emerged as promising biomarkers of disease status especially for metastatic cancer. ${ }^{5-7}$ Previous reports have shown that ctDNA derived from plasma cell-free DNA carries genetic information from tumor genome that can indicate one's disease status at certain circumstances. ${ }^{8}{ }^{8}$ In melanoma, several studies have shown that using ctDNA is capable of monitoring treatment response and identifying new resistance mechanisms in patients with BRAF mutant tumors. ${ }^{8,10-15}$

In parallel to the CT scanning, the patient was monitored for disease status by NGS-based ctDNA sequencing. Mutations with clinical interpretations including BRAF V600E, TP53 V73 frameshift and PIK3CA H450 P458 deletion were called at baseline prior to the initiating chemotherapy in his NGS report. Other genomic alterations emerged as tumor evolution, such as MTOR T1977R. Dynamic changes of the mutation fractions in the plasma samples were shown in Figure 4. His ctDNA fractions significantly increased at April 2019, which provided an early indication of disease progression. On the other side, the tumor markers (CEA and CA19-9) were not elevated until June 2019 (Figure 2), when the patient was confirmed PD through CT imaging. Therefore, this case highlights the role of ctDNA monitoring in advanced CRC. Targeting tumor-related mutations could inform treatment response as well as the disease status ahead of time. Studies have shown that ctDNA can be reliably detected in early stages CRC patients. ${ }^{16,17}$ That means low levels of ctDNA fractions are technically detectable, thus, plasma ctDNA may be useful in identifying new developing lesions, and providing potential treatment options.

Advanced CRC with BRAF V600E mutation is associated with poor prognosis. Aggressive combination therapy is required to improve the survival rate. ${ }^{18}$ Several treatment strategies have been approved or under investigation for BRAF V600E positive mCRC. Current NCCN guideline recommended encorafenib plus cetuximab/panitumumab in second-line setting treatment of BRAF V600E 


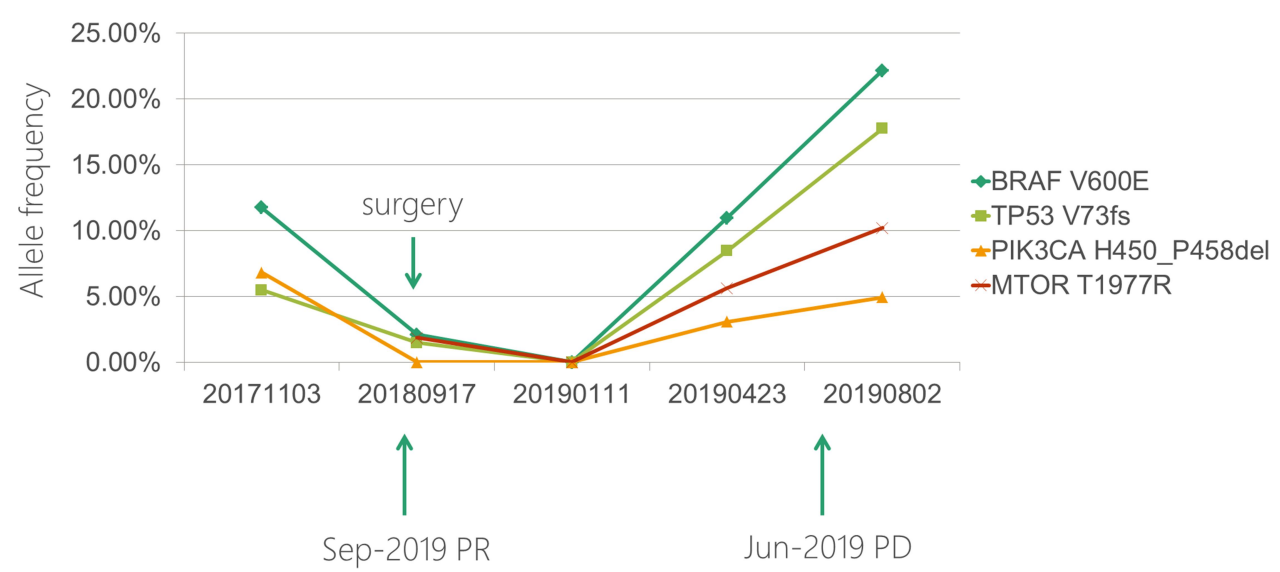

Figure 4 Dynamic changes of genetic mutation fractions in ctDNA.

positive mCRC. Moreover, combination therapy including BRAF, EGFR and MEK inhibitors was reported with $41 \%$ ORR in mCRC patients with BRAF V600E mutations in the BEACON trial (NCT02928224, Phase 3 study in patients with BRAFV600E mCRC whose disease has progressed after 1 or 2 prior regimens in the metastatic setting). ${ }^{19}$ However, some of these drugs have not been approved in China. The SWOG 1406 study evaluated vemurafenib in combination with cetuximab and irinotecan in patient with BRAF V600E mutant mCRC after 1 or 2 prior regimens with no prior anti-EGFR agents. The addition of vemurafenib achieved higher PFS (4.4 mo vs $2 \mathrm{mo}$ ) and disease control rates $(67 \%) .{ }^{20,21}$ Such combination therapy could be a feasible treatment for Chinese mCRC patients with BRAF mutations. Previously we reported a patient with BRAF V600E positive colon adenocarcinoma who showed complete tumor recession under treatment of irinotecan, cetuximab, vemurafenib and $5-\mathrm{Fu}^{22} \mathrm{~A}$ clinical trial is ongoing investigating the efficacy of FIVC strategy in advanced CRC patients with BRAF V600E mutation. The patient in the present case was enrolled in the trial (FIVC, NCT03727763), since he is a young man in good physical condition.

REGONIVO is a study investigating the safety and efficacy of regorafenib plus nivolumab in previously treated patients with advanced gastric cancer or CRC. Seven of 25 CRC patients showed objective response. ${ }^{23}$ The overall disease control rate was $88 \%$, with a median 6.3 months PFS. No CRC patients with BRAF mutation were reported in REGONIVO study. The same clinical trial is ongoing in our hospital [NCT04110093] investigating the efficacy and safety of the combination of regorafenib and nivolumab as third-line or later-line therapy in patients with MSS mCRC. Patient in the present case has received REGONIVO treatment for more than 12 months by late August 2020 with remission of his liver lesion. His best response was PR. He is still on-treatment.

In summary, we report treatment of a mCRC patient with genomic BRAF V600E alteration. The patient achieved R0 resection after several cycles of combination treatment of vemurafenib, cetuximab, 5-fluorouracil and irinotecan. Sequencing of ctDNA for tumor-related mutations during treatment could indicate disease status and provide useful information for further treatment. Interestingly, he achieved PR in the subsequent treatment of REGONIVO with more than 12-month PFS.

\section{Ethics and Consent Statement}

Written informed consent was obtained from the study participant prior to study commencement.

\section{Consent for Publication}

Written informed consent was obtained from the patient for publication of this case report.

\section{Acknowledgments}

The authors would like to thank the patient for giving consent. Zhan Wang and Chen-Yang Ye are co-first authors for this study.

\section{Disclosure}

Jingjing Zheng is an employee of Burning Rock Biotech. The authors report no other conflicts of interest in this work.

\section{References}

1. Tárraga López PJ, Albero JS, Rodríguez-Montes JA. Primary and secondary prevention of colorectal cancer. Clin Med Insights Gastroenterol. 2014;7:33-46. 
2. Yokota T, Ura T, Shibata N, et al. BRAF mutation is a powerful prognostic factor in advanced and recurrent colorectal cancer. $\mathrm{Br}$ $J$ Cancer. 2011;104(5):856-862. doi:10.1038/bjc.2011.19

3. Morikawa T, Inada R, Nagasaka T, et al. BRAF V600E mutation is a predictive indicator of upfront chemotherapy for stage IV colorectal cancer. Oncol Lett. 2018;15(2):2195-2201.

4. Bahrami A, Hesari A, Khazaei M, et al. The therapeutic potential of targeting the BRAF mutation in patients with colorectal cancer. $J$ Cell Physiol. 2018;233(3):2162-2169. doi:10.1002/jcp.25952

5. Calapre L, Warburton L, Millward M, et al. Circulating tumour DNA (ctDNA) as a liquid biopsy for melanoma. Cancer Lett. 2017;404:62-69. doi:10.1016/j.canlet.2017.06.030

6. Wan J, Massie C, Garcia-Corbacho J, et al. Liquid biopsies come of age: towards implementation of circulating tumour DNA. Nat Rev Cancer. 2017;17(4):223-238.

7. Heitzer E, Ulz P, Geigl JB. Circulating tumor DNA as a liquid biopsy for cancer. Clin Chem. 2015;61(1):112-123. doi:10.1373/ clinchem.2014.222679

8. Stephen Q, Wong JMR, Jason Callahan IAV, et al. Circulating tumor DNA analysis and functional imaging provide complementary approaches for comprehensive disease monitoring in metastatic melanoma. JCO Precis Oncol. 2017;1:1-14.

9. Murtaza M, Dawson SJ, Pogrebniak K, et al. Multifocal clonal evolution characterized using circulating tumour DNA in a case of metastatic breast cancer. Nat Commun. 2015;6:8760. doi:10.1038/ ncomms 9760

10. Gray ES, Rizos H, Reid AL, et al. Circulating tumor DNA to monitor treatment response and detect acquired resistance in patients with metastatic melanoma. Oncotarget. 2015;6(39):42008-42018. doi:10.18632/oncotarget.5788

11. Sanmamed MF, Fernández-Landázuri S, Rodríguez $\mathrm{C}$, et al. Quantitative cell-free circulating BRAFV600E mutation analysis by use of droplet digital PCR in the follow-up of patients with melanoma being treated with BRAF inhibitors. Clin Chem. 2015;61 (1):297-304. doi:10.1373/clinchem.2014.230235

12. Girotti MR, Gremel G, Lee R, et al. Application of sequencing, liquid biopsies, and patient-derived xenografts for personalized medicine in melanoma. Cancer Discov. 2016;6(3):286-299. doi:10.1158/2159. 8290.CD-15-1336

13. Gonzalez-Cao M, Mayo-de-Las-Casas C, Molina-Vila MA, et al. BRAF mutation analysis in circulating free tumor DNA of melanoma patients treated with BRAF inhibitors. Melanoma Res. 2015;25 (6):486-495. doi:10.1097/CMR.0000000000000187
14. Schreuer M, Meersseman G, Van Den Herrewegen S, et al. Quantitative assessment of BRAF V600 mutant circulating cell-free tumor DNA as a tool for therapeutic monitoring in metastatic melanoma patients treated with BRAF/MEK inhibitors. J Transl Med. 2016;14:95. doi:10.1186/s12967-016-0852-6

15. Lee JH, Long GV, Boyd S, et al. Circulating tumour DNA predicts response to anti-PD1 antibodies in metastatic melanoma. Ann Oncol. 2017;28(5):1130-1136. doi:10.1093/annonc/mdx026

16. Bettegowda C, Sausen M, Leary RJ, et al. Detection of circulating tumor DNA in early- and late-stage human malignancies. Sci Transl Med. 2014;6(224):224ra24. doi:10.1126/scitranslmed.3007094

17. Tie J, Wang Y, Tomasetti C, et al. Circulating tumor DNA analysis detects minimal residual disease and predicts recurrence in patients with stage II colon cancer. Sci Transl Med. 2016;8(346):346ra92. doi:10.1126/scitranslmed.aaf6219

18. Strickler JH, Wu C, Bekaii-Saab T. Targeting BRAF in metastatic colorectal cancer: maximizing molecular approaches. Cancer Treat Rev. 2017;60:109-119. doi:10.1016/j.ctrv.2017.08.006

19. Van Cutsem E, Huijberts S, Grothey A, et al. Binimetinib, encorafenib, and cetuximab triplet therapy for patients with BRAF V600E-mutant metastatic colorectal cancer: safety lead-in results from the phase III BEACON colorectal cancer study. J Clin Oncol. 2019;37(17):1460-1469. doi:10.1200/JCO.18.02459

20. Hong DS, Morris VK, El Osta B, et al. Phase IB study of vemurafenib in combination with irinotecan and cetuximab in patients with metastatic colorectal cancer with BRAFV600E mutation. Cancer Discov. 2016;6(12):1352-1365. doi:10.1158/2159-8290.CD-16-0050

21. Kopetz S, Desai J, Chan E, et al. Phase II pilot study of vemurafenib in patients with metastatic BRAF-mutated colorectal cancer. J Clin Oncol. 2015;33(34):4032-4038. doi:10.1200/JCO.2015.63.2497

22. Wang Z, Dai WP, Zang YS. Complete response with fluorouracil and irinotecan with a BRAFV600E and EGFR inhibitor in BRAF-mutated metastatic colorectal cancer: a case report. Onco Targets Ther. 2019;12:443-447. doi:10.2147/OTT.S180845

23. Fukuoka S, Hara H, Takahashi N, et al. Regorafenib plus nivolumab in patients with advanced gastric (GC) or colorectal cancer (CRC): an open-label, dose-finding, and dose-expansion phase $1 \mathrm{~b}$ trial (REGONIVO, EPOC1603). 2019:2522.
OncoTargets and Therapy

\section{Publish your work in this journal}

OncoTargets and Therapy is an international, peer-reviewed, open access journal focusing on the pathological basis of all cancers, potential targets for therapy and treatment protocols employed to improve the management of cancer patients. The journal also focuses on the impact of management programs and new therapeutic agents and protocols on patient perspectives such as quality of life, adherence and satisfaction. The manuscript management system is completely online and includes a very quick and fair peer-review system, which is all easy to use. Visit http://www.dovepress.com/ testimonials.php to read real quotes from published authors. 